\title{
Cpg-ODN, a TLR9 Agonist, Aggravates Myocardial Ischemia/Reperfusion Injury by Activation of TLR9-P38 MAPK Signaling
}

\author{
Liang Xie Songqing He Na Kong Ying Zhu Yi Tang Jianhua Li \\ Zhengbing Liu Jing Liu Jianbin Gong \\ Department of Cardiology, Jinling Hospital, Nanjing University School of Medicine, Nanjing, Jiangsu, \\ China
}

\section{Key Words}

Cpg-ODN • Ischemia/reperfusion injury• TLR9-p38 MAPK signaling

\begin{abstract}
Background/Aims: Toll-like receptors (TLRs) have been implicated in myocardial ischemia/ reperfusion (I/R) injury. We examined the effect of CpG-oligodeoxynucleotide (ODN) on myocardial I/R injury. Methods: Male Sprague-Dawley rats were treated with either CpG-ODN or control ODN $1 \mathrm{~h}$ prior to myocardial ischemia (30 min) followed by reperfusion. Rats treated with phosphate-buffered saline (PBS) served as I/R controls ( $n=8 /$ group). Infarct size was determined by 2,3,5-triphenyltetrazolium chloride and Evans blue straining. Cardiac function was examined by echocardiography before and up to 14 days after myocardial I/R. Results: CpG-ODN administration significantly increased infarct size and reduced cardiac function and survival rate after myocardial I/R, compared to the PBS-treated I/R group. ControlODN did not alter I/R-induced myocardial infarct size, cardiac dysfunction, and survival rate. Additionally, CpG-ODN promoted I/R-induced myocardial apoptosis and cleaved caspase-3 levels in the myocardium. CPG-ODN increased TLR9 activation and p38 phosphorylation in the myocardium. In vitro data also suggested that CPG-ODN treatment induced TLR9 activation and p38 phosphorylation. Importantly, p38 mitogen-activated protein kinase (MAPK) inhibition abolished CpG-ODN-induced cardiac injury. Conclusion: CpG-ODN, the TLR9 ligand, accelerates myocardial I/R injury. The mechanisms involve activation of the TLR9-p38 MAPK signaling pathway.

\section{Introduction}

The Toll-like receptor (TLR) family now consists of 10 members in humans (TLR1TLR10) and 12 members in mice (TLR1-TLR9, TLR11-TLR13), which function to recognize pathogen-associated molecular patterns (PAMPs) and trigger immune responses in the L. Xie and S. He contributed equally to this work.

Jing Liu, PhD

and Jianbin Gong, MD 
body. TLRs play a crucial role in inducing innate immune and inflammatory responses [1]. Upon PAMP ligand binding, TLRs trigger a cascade of molecular events, leading to activation of NF- $\kappa B$ signaling and expression of downstream targets. TLRs can induce p38 mitogenactivated protein kinase (MAPK) signaling as well [2]. A large body of evidence shows that p38 plays a central role in regulating many cellular processes, including inflammation, cell cycle progression, differentiation, and apoptosis. TLR9 is special in that it is localized in endolysosomes and has a unique ligand, the unmethylated CpG-DNA from bacteria and endogenous DNA [3, 4]. CpG motifs can activate the TLR9-mediated NF- $\kappa B$ signaling pathway and MAPKs [4]. Synthetic CpG-oligodeoxynucleotide (ODN) has been shown to activate TLR9 [4-6]. Recently, mitochondrial DNA (mtDNA), which is also unmethylated at CpG motifs, has been shown to be taken up by immune cells in which it induces an inflammatory response through activating TLR9 [7]. Innate immunity and inflammatory responses are also associated with myocardial ischemia/reperfusion (I/R) injuries [8-11].

Several studies have reported a benefit of CpG-ODN in sepsis and cardiac function after I/R [12-15]. However, recent studies have demonstrated that TLR9 could be a therapeutic target for reducing myocardial I/R injury. Zhou et al. have shown that inhibitory CpGODN (iCpG-ODN) induced protection against myocardial I/R via inhibiting inflammatory responses in a dose-dependent manner and may be useful in therapy for stroke patients [16]. Shaker et al. also reported that TLR9 signaling inhibition by COV08-0064 may be an effective approach in liver surgeries, including transplantation, to limit I/R injury [17]. Our previous study found that intravenous injection of mtDNA into rats aggravated I/R injury and increased infarct area through TLR9-p38 MAPK activation [18]. Similarly to mtDNA, CpG-ODN can also activate TLR9.

In the present study, we examined the effect of CpG-ODN on myocardial I/R injury. We hypothesized that $\mathrm{CpG}-\mathrm{ODN}$ induces an innate immune response and aggravates I/R injury possibly via the TLR9-p38 MAPK pathway. To test this hypothesis, we examined the effect of CpG-ODN on rat H9c2s cells in vitro and on myocardium using the rat model of I/R injury.

\section{Materials and Methods}

\section{Reagents}

Mouse anti-TLR9 antibody was obtained from Abcam (Cambridge, MA). Antibodies against p38 MAPK, phospho-p38 MAPK (Thr180/Tyr182), and caspase-3 were purchased from Cell Signaling Technology (Danvers, MA). Goat anti-mouse IgG-HRP and goat anti-rabbit IgG-HRP were obtained from Boster Biotechnology Co., Ltd. (Wuhan, China). The p38 MAPK inhibitor SB203580 was purchased from Calbiochem (San Diego, CA). The CpG-ODN (CpG-ODN 1826) and control ODN (control-ODN 1826) were purchased from InvivoGen (San Diego, CA).

\section{Animals}

Male Sprague-Dawley rats (aged ca. 4 months, 300-350 g) were used in these studies and were obtained from the Comparative Medicine Department of Jinling Hospital. The animal care and experimental procedures were performed in compliance with the Institutional Animal Care and User Guidelines and were approved by the Comparative Medicine Department of Jinling Hospital.

\section{Establishment of I/R model}

Myocardial I/R injury was induced as described previously [19-21]. Briefly, the rats were anesthetized by $5.0 \%$ isoflurane, intubated, and ventilated with room air using a rodent ventilator. Body temperature was maintained at $37^{\circ} \mathrm{C}$ by surface water heating. Following the skin incision, the hearts were exposed through a left thoracotomy in the fourth intercostal space. The left anterior descending (LAD) coronary artery was ligated with a 7-0 silk ligature over a 1-mm polyethylene tube (PE-10). After completion of $30 \mathrm{~min}$ of occlusion, the coronary artery was reperfused by pulling on the exteriorized suture to release the knot. The sham-operated animals underwent the same surgical procedures except that the suture around the LAD was not fastened. The skin was closed and the animals were allowed to recover in prewarmed cages. 


\section{Cellular Physiology Cell Physiol Biochem 2018;47:1389-1398

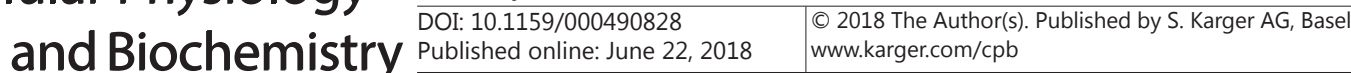 \\ Xie et al.: Cpg-ODN Aggravates Myocardial Ischemia/Reperfusion Injury}

\section{Rat experiment protocol}

The rats were treated with CpG-ODN ( $n=8,3 \mathrm{mg} / \mathrm{kg}$ body weight), control-ODN ( $n=8,3 \mathrm{mg} / \mathrm{kg}$ body weight), or phosphate buffered saline (PBS; $n=8$ ) by intraperitoneal (i.p.) injection $1 \mathrm{~h}$ before the hearts were subjected to I/R. We also examined the effect of CpG-ODN on cardiac function following myocardial I/R injury. The rats ( $n=8$ per group) were treated with CpG-ODN, control-ODN, or PBS $1 \mathrm{~h}$ before the hearts were subjected to $30 \mathrm{~min}$ of ischemia followed by reperfusion up to 14 days. Cardiac function was examined by echocardiography before I/R, and 7 and 14 days after reperfusion as described previously [22, 23]. To determine the role of the TLR9-p38 signaling pathway in TLR9-ligand-induced myocardial injury, the rats $(n=8)$ were injected with the p38 inhibitor SB203580 (10 mg/kg body weight) $20 \mathrm{~min}$ prior to CpG-ODN administration ( $3 \mathrm{mg} / \mathrm{kg}$ body weight).

\section{Cell culture and treatment}

The rat H9c2 cardiomyocyte cell line, obtained from American Type Culture Collection (ATCC; Rockville, MD), was cultured in Dulbecco's modified Eagle's medium supplemented with $10 \%$ fetal bovine serum, antibiotics (50 U/ml penicillin and $50 \mu \mathrm{g} / \mathrm{ml}$ streptomycin) at $37^{\circ} \mathrm{C}$ in an atmosphere of $95 \%$ air and $5 \%$ $\mathrm{CO}_{2}$. The medium was replaced every 2 or 3 days and the cells were subjected to experimental procedures at $80-90 \%$ confluence. H9c2 cells were treated with CpG-ODN (100 nM) or control-ODN (100 nM), in the presence and absence of SB203580 (15 $\mu \mathrm{M})$ for $20 \mathrm{~min}$. Each group contained three replicates. The cells were harvested and cellular proteins were prepared for western blot analysis as described previously [22].

\section{Echocardiography}

Serial echocardiographic studies were performed as described under light anesthesia (pentobarbital, $25 \mathrm{mg} / \mathrm{kg}$, i.p.) 1 day before surgery and at 7 days and 14 days after I/R treatment. The anterior chest was shaved and rats were placed in the left lateral decubitus position. Body temperature was maintained between $36.9^{\circ} \mathrm{C}$ and $37.3^{\circ} \mathrm{C}$. Echocardiographic images were obtained using an HP SONOS 7500 ultrasound system equipped with a L12-5 linear broadband and a S12 phased array transducer fitted with a 0.3-cm standoff. The heart was imaged in the parasternal short axis view at the level of the papillary muscles to determine ejection fraction (EF). All measurements were averaged in three consecutive cardiac cycles and analyzed off-line by a single blinded observer using COMPACS image analysis software. All calculations were derived using standard formulas. Left ventricular end-diastolic diameters (LVDd) were measured from M-mode tracings obtained at the mid-papillary level and analyzed according to modified American Society for Echocardiography standards (posterior wall leading edge-to-leading edge and anterior wall trailing edge-to-trailing edge).

\section{Western blot analysis}

The H9c2s cells and the rat whole heart tissues in each group were disrupted in lysis buffer $(20 \mathrm{mM}$ Tris pH 7.5, $150 \mathrm{mM} \mathrm{NaCl}, 1 \%$ Triton X-100, $2.5 \mathrm{mM}$ sodium pyrophosphate, $1 \mathrm{mM}$ EDTA, 1\% $\mathrm{Na}_{3} \mathrm{VO}_{4}$, $0.5 \mu \mathrm{g} / \mathrm{ml}$ leupeptin, $1 \mathrm{mM}$ phenylmethanesulfonyl fluoride), and the cell lysates were centrifuged at 12, $000 \times g$ at $4^{\circ} \mathrm{C}$ for $5 \mathrm{~min}$. Protein concentrations of the supernatants were determined by a bicinchoninic acid protein assay kit (Beyotime Institute of Biotechnology, Jiangsu, China). We performed a western blot analysis of the cellular and tissue lysates as previously described [24]. After denaturation at $100^{\circ} \mathrm{C}$ for $5 \mathrm{~min}$ in loading buffer (Bio-Rad), equivalent amounts of protein $(10 \mu \mathrm{g})$ were separated by electrophoresis in $10 \%$ sodium dodecyl sulfate polyacrylamide gel electrophoresis gels and electrophoretically transferred to a polyvinylidene difluoride membrane for $90 \mathrm{~min}$ at $100 \mathrm{~V}$. After blocking membranes with 5\% milk in TBST (50 mM Tris- $\mathrm{HCl}, \mathrm{pH} 8 ; 154 \mathrm{mM} \mathrm{NaCl}$ and $0.1 \%$ Tween 20 ) for $2 \mathrm{~h}$ at room temperature, membranes were incubated overnight at $4^{\circ} \mathrm{C}$ with the respective antibodies. A chemiluminescence detection kit was used to detect the signals on the blots. Densitometry was performed on the X-ray films that were exposed to the blots using the ChemiDoc system (UVP, Upland, CA).

\section{Measurement of infarct zone and risk area}

The LAD coronary artery was re-occluded with a 7-0 Prolene suture that was used previously at the same place for rats assigned for measurement of infarct zone and risk area, and Evans blue dye ( $2 \mathrm{ml}$ of a $1 \%$ solution) was injected via the external jugular vein to delineate the area at risk (AAR). The rats were sacrificed under deep pentobarbital anesthesia (60 mg/kg, i.p.) after blood sampling. The heart was then 


\section{Cellular Physiology Cell Physiol Biochem 2018;47:1389-1398 \begin{tabular}{l|l} 
and Biochemistry Published online: June 22, 2018 & $\begin{array}{l}\text { D) } 2018 \text { The Author(s). Published by S. Karger AG, Basel } \\
\text { www.karger.com/cpb }\end{array}$ \\
\hline
\end{tabular} Xie et al.: Cpg-ODN Aggravates Myocardial Ischemia/Reperfusion Injury}

rapidly excised and washed in $0.9 \%$ saline. After removal of the atrium, the ventricle was cut into transverse slices of equal thickness $(3 \mathrm{~mm}$ ) from the apex to the base. The slices were then incubated for $20 \mathrm{~min}$ in phosphate-buffered $1 \%$ 2, 3,5-triphenyltetrazolium chloride (TTC) at $37^{\circ} \mathrm{C}$, and then fixed in $10 \%$ formalin solution. The AAR was defined as the area not stained with Evans blue dye. The area not stained by TTC was defined as the infarcted zone (AI). The border zones (Evans blue-stained area neighboring Evans blueunstained area), infarcted zones (TTC and border zones [TTC-stained], infarcted zones [TTC and Evans blueunstained]), and the nonischemic zones (Evans blue-unstained area remote from Evans blue-unstained area) were photographed and analyzed by the software program ImageJ 1.36 (National Institutes of Health $[\mathrm{NIH}]$, Bethesda, MD). The AAR, AI, and ventricle size were assessed by a technician who was blinded to the experimental protocol using computer-assisted planimetry (NIH ImageJ 1.57 software).

\section{Terminal deoxynucleotidyl transferase-mediated dUTP nick end labeling}

We examined whether CpG-ODN would lead to increased apoptosis in LV myocardium, by terminal deoxynucleotidyl transferase-mediated dUTP nick end labeling (TUNEL) staining. TUNEL staining was performed on the heart sections using TdT-FragEL apoptosis detection kits (Calbiochem) according to the manufacturer's instructions. Counterstaining with methyl green was performed to distinguish between normal and apoptotic nuclei, in which normal nuclei were stained as blue and apoptotic nuclei as brown. The number of TUNEL-positive cells within a $2.5-\mathrm{mm}^{2}$ field in the LV free wall was counted. Eight randomly selected fields per section and five sections per heart were examined and the counts were averaged for statistical analysis. The observer was blinded to the treatment groups.

\section{Statistical analysis}

The study data are expressed as the mean \pm standard deviation (SD) and were assessed for statistical significance using Student's $t$ test. Results of the treatment groups were compared to those of the control group using one-way analysis of variance followed by Dunnett's test. All of the calculations and statistical analyses were performed using SPSS software for Windows (version 22.0; SPSS Corp., Chicago, IL). Rat survival curves were constructed using the Kaplan-Meier method within Prism (GraphPad Software, San Diego, CA).

\section{Results}

CpG-ODN administration increased myocardial infarction and reduced survival rate following $I / R$

To investigate the effect of the TLR9 ligand CpG-ODN on myocardial infarction following $\mathrm{I} / \mathrm{R}$, we administered $\mathrm{CpG}-\mathrm{ODN}$ to rats $1 \mathrm{~h}$ prior to myocardial ischemia (30 min) followed by reperfusion $(2 \mathrm{~h})$. Fig. $1 \mathrm{~A}$ shows that $\mathrm{CpG}-\mathrm{ODN}$ treatment significantly increased infarct size compared with PBS-treated I/R hearts $(50.66 \% \pm 5.10 \%$ vs. $20.99 \% \pm 4.11 \%)$. There was no significant difference in the ratio of risk area/LV area between CpG-ODN-treated rats and PBS-treated I/R rats, suggesting that the coronary artery was ligated in the same place in the groups (Fig. 1A). Administration of control-ODN to the rats did not alter I/R-induced myocardial infarction. We also evaluated the effect of CpG-ODN administration on survival rate following myocardial I/R. As shown in Fig. 2B, in PBS-treated rats, 93.3\% of rats survived at $24 \mathrm{~h}$ and $70.9 \%$ survived 30 days after I/R. In CpG-ODN-treated rats, however, $13.3 \%$ of rats died in the first $24 \mathrm{~h}$ and $60.0 \%$ died 10 days following myocardial I/R. There was no significant difference in the survival rate between control-ODN-treated and PBS-treated I/R groups.

CpG-ODN administration significantly reduced cardiac function after myocardial $I / R$

We also examined the effect of CpG-ODN on cardiac function following myocardial I/R. Fig. 2A shows representative M-mode echocardiographic images from a PBS-treated and CpG-ODN-treated rat recorded 7 days after I/R. Fig. 2B and 2C show that EF and fractional shortening values in CpG-ODN-treated rats were significantly decreased by $62.3 \%$ and $34.6 \%$ at 7 days, and by $56.6 \%$ and $36.4 \%$ at 14 days after reperfusion, respectively, compared with 
untreated I/R rats. LVDd in CpG-ODN-treated rats was significantly increased by $50.4 \%$ at 7 days and $72.9 \%$ at 14 days after reperfusion. Treatment of rats with control-ODN did not affect I/R-induced cardiac dysfunction. In contrast, CpG-ODN administration aggravated I/R-induced cardiac dysfunction. Fig. 2D shows that LVDd was significantly increased after myocardial ischemia followed by reperfusion up to 14 days.

CPG-ODN administration aggravated I/R-increased myocardial apoptosis

It is well known that myocardial apoptosis contributes to myocardial ischemic injury [25]. We examined the effect of CpG-ODN treatment on I/R-induced myocardial apoptosis.

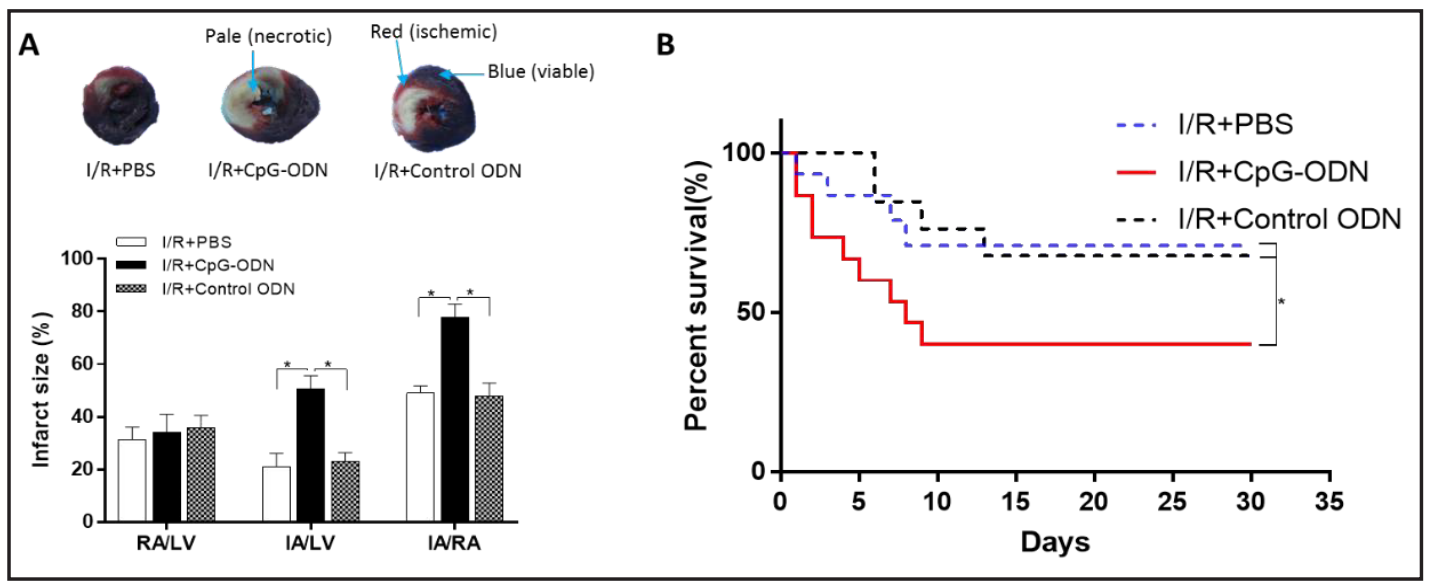

Fig. 1. (A) The TLR9 ligand CPG-ODN increased myocardial infarct size following I/R injury. The rats were treated with PBS, CpG-ODN, or control CpG-ODN ( $n=8$ rats per group) by i.p. injection $1 \mathrm{~h}$ prior to myocardial ischemia followed by reperfusion ( $2 \mathrm{~h}$ ). The infarct area (white) and the area at risk (red+white) from each section were measured using an image analyzer. Ratios of risk area vs. left ventricle area (RA/LV) and infarct area vs. risk area (IA/RA) were calculated and are presented in the graph. ${ }^{*} \mathrm{P}<0.05$ compared with the indicated group. (B) Survival rate was evaluated following myocardial I/R ( $=15$ per group). ${ }^{*} \mathrm{P}<0.05$ compared with the indicated group. I/R, ischemia/reperfusion; ODN, oligodeoxynucleotide; PBS, phosphate buffered saline.

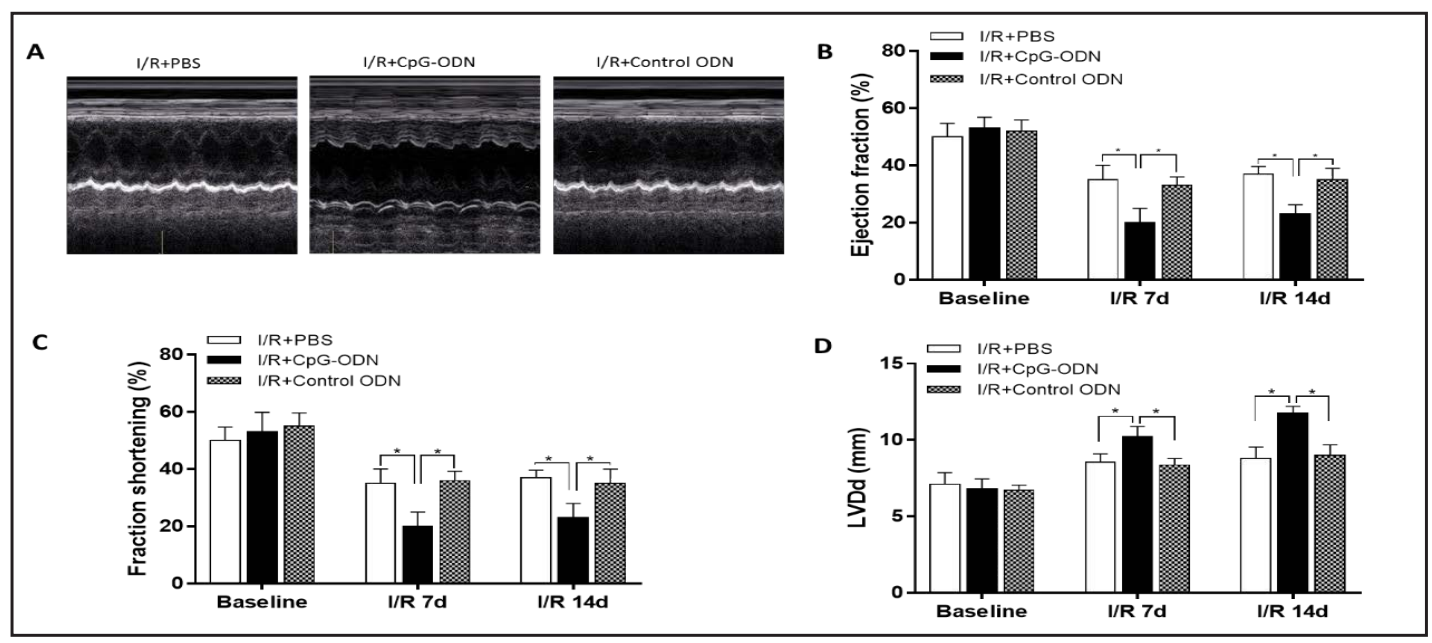

Fig. 2. Echocardiographic assessment of cardiac function. (A) Representative M-mode echocardiographic images from a PBS-, CpG-ODN-, and control-ODN-treated rat recorded 7 days after I/R. (B) Quantitative analysis of ejection fraction of each group recorded before I/R (baseline), and 7 days and 14 days after I/R. (C) Quantitative analysis of fractional shortening of each group. (D) Quantitative analysis of left ventricular end-diastolic diameter (LVDd) of each group. $\mathrm{n}=8$ rats per group. ${ }^{*} \mathrm{P}<0.05$ compared with the indicated group. I/R, ischemia/reperfusion; ODN, oligodeoxynucleotide; PBS, phosphate buffered saline. 
Fig. 3A and 3B show that I/R markedly induced myocardial apoptosis, as well as a 95\% increase in the number of apoptotic cells in CpG-ODN-treated I/R rats compared with PBStreated I/R hearts ( $n=6$ per group). Control-ODN administration did not affect I/R-induced apoptosis in the myocardium (Fig. 3A). We harvested the heart after 120 min of reperfusion. Fig. 3B shows an increased level of cleaved caspase-3 in CpG-ODN-treated I/R hearts $(n=6)$. The levels of the proteins were quantified using densitometry and normalized to GAPDH. Data are presented as the mean $\pm \mathrm{SD}$. These experiments were performed in quintuplicate with similar results.

CpG-ODN administration increases levels of TLR9 and p38 phosphorylation in the heart tissues following myocardial $I / R$

We examined whether CpG-ODN administration induces the activation of TLR9-p38 MAPK signaling using the myocardium at risk following I/R. Fig. 4 shows that CpG-ODN treatment significantly increased the levels of TLR9 and phospho-p38 compared with the untreated I/R group. Control-ODN administration did not significantly alter the levels of phospho-p38 or TLR9 in the myocardium of untreated sham and I/R rats. Treatment of rats with the specific p38 inhibitor SB203580 abolished CpG-ODN-increased levels of phospho-p38 in the myocardium.

CPG-ODN administration increases levels of TLR9 and p38 phosphorylation in H9c2 cells

To explore the mechanism of myocardial damage induced by CpG-ODN, we tested whether CpG-ODN can activate the TLR9-p38 MAPK pathway in cultured H9c2 cells. H9c2 cells were incubated with CpG-ODN with or without SB203580, followed by western blotting assay to examine the activation of TLR9-p38 MAPK signaling. As shown in Fig. 5A and 5B, CpG-ODN exposure induced significant upregulation of TLR9 protein and phosphorylated p38 MAPK, and the effect of CpG-ODN was weakened by SB203580 pretreatment. In these treatments, the levels of total p38 MAPK were not significantly changed. These results suggested that CpG-ODN activates the TLR9-p38 MAPK pathway in cultured H9c2 cells.

p38 MAPK inhibition abolished CPG-ODNinduced cardiac injury

To determine the role of the TLR9-p38 MAPK signaling pathway in CpGODN-induced cardiac injury, we treated the rats with the p38 inhibitor SB203580 20 min prior to CpG-ODN administration. The hearts were subjected to ischemia (30 $\mathrm{min})$ followed by reperfusion $(2$ h). Fig. 6 shows that SB203580 administration abolished CpG-ODNinduced cardiac injury.

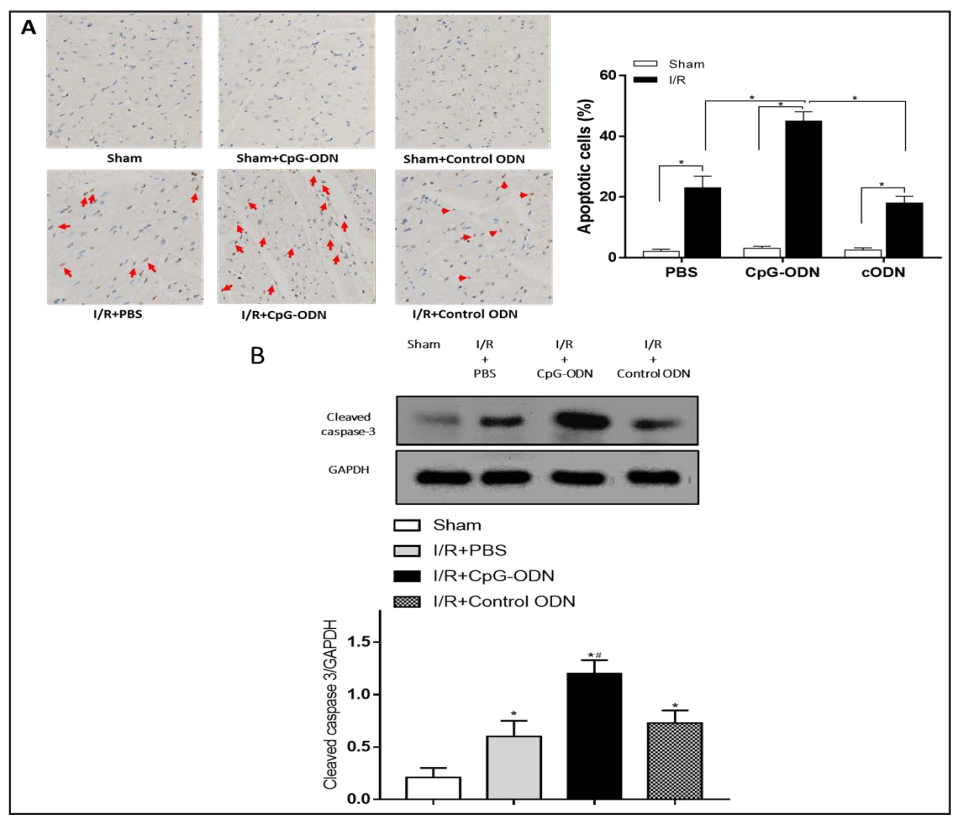

Fig. 3. Apoptosis in the myocardium. (A) Terminal deoxynucleotidyl transferase-mediated dUTP nick end labeling staining for apoptosis in the nonischemic and ischemic myocardium. Brown spots (arrows) in nuclei indicate cell death. (B) The level of cleaved caspase-3 protein in rat hearts treated with PBS, CpG-ODN, or control CpG-ODN. $\mathrm{n}=6$ rats per group. ${ }^{*} \mathrm{P}<0.05$ compared with the indicated group. $\# \mathrm{P}<0.05$ vs. * $\mathrm{I} / \mathrm{R}$, ischemia/reperfusion; ODN, oligodeoxynucleotide; PBS, phosphate buffered saline. 


\section{Cellular Physiology and Biochemistry}

Cell Physiol Biochem 2018;47:1389-1398

\begin{tabular}{l|l}
\hline DOI: $10.1159 / 000490828$ & (c) 2018 The Author(s). Published by S. Karger AG, Basel
\end{tabular}

Xie et al.: Cpg-ODN Aggravates Myocardial Ischemia/Reperfusion Injury
Infarct size in CpG-ODNtreated rats in the presence of SB203580 was significantly decreased compared with CpG-ODN-treated rats that did not receive SB203580 $(21.71 \% \pm 3.00 \%$ vs. $51.66 \%$ $\pm 5.12 \%$ ). There was no significant difference in the infarct size between the CpGODN+SB203580 group and the PBS-treated I/R group.

\section{Discussion}

A major finding in this study is that administration of a TLR9 agonist, CpG-ODN, to rats increased infarct size and reduced cardiac function after myocardial $\mathrm{I} / \mathrm{R}$ injury. Administration of control CpG-ODN did not affect I/R-induced myocardial infarction, suggesting that activation of TLR9 plays a traumatic role during myocardial I/R injury. More significantly, CpG-ODN treatment activated the TLR9-p38 MAPK signaling pathway. The data suggest that the TLR9 agonist CpGODN promotes myocardial injury involving activation of the TLR9-p38 MAPK signaling pathway. The data also indicate that the apoptosis-promoting effect of CpG-ODN may be one of the mechanisms by which CpGODN aggravates myocardial I/R injury.

$$
\text { Cardiac myocyte }
$$
apoptosis plays a role in myocardial I/R injury. We have previously reported that mtDNA activates TLR9-p38 MAPK signaling and promotes myocardial I/R-induced myocardial injury and cardial apoptosis [18]. Both mtDNA and CpG-

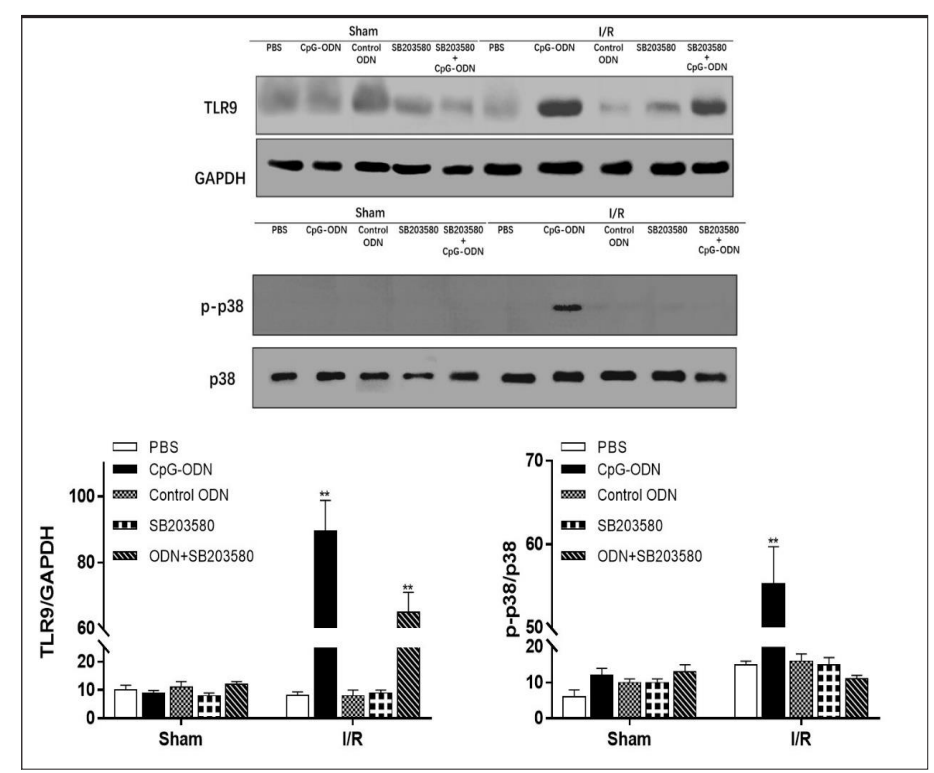

Fig. 4. CpG-ODN administration increases levels of TLR9 and p38 phosphorylation in heart tissues. The level of TLR9 protein in hearts treated with PBS, CpG-ODN, control CpG-ODN, SB203580, or CpGODN+SB203580 prior to I/R. Total heart proteins were analyzed by sodium dodecyl sulfate polyacrylamide gel electrophoresis and immunoblotted for TLR9; GAPDH served as a loading control. ${ }^{* *} \mathrm{P}<0.01$, $\mathrm{n}=3$. The level of p38 and phosphorylated p38 (p-p38) protein in hearts treated with PBS, CpG-ODN, control CpG-ODN, SB203580, or CpG-ODN+SB203580 prior to I/R. ${ }^{* *} \mathrm{P}<0.01, \mathrm{n}=3$. I/R, ischemia/ reperfusion; ODN, oligodeoxynucleotide; PBS, phosphate buffered saline.

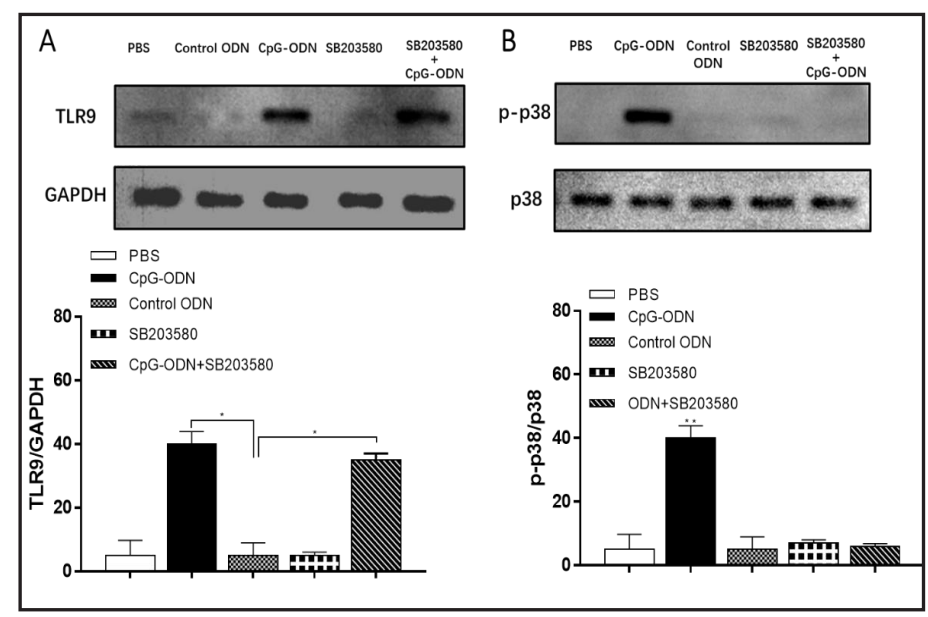

Fig. 5. CpG-ODN administration increases levels of TLR9 and p38 phosphorylation in cultured H9c2 cells. (A) Level of TLR9 protein in H9c2s treated with PBS, CpG-ODN, control CpG-ODN, SB203580, or CpG-ODN+SB203580 prior to I/R. GAPDH served as a loading control. ${ }^{*} \mathrm{P}<0.05,{ }^{* *} \mathrm{P}<0.01, \mathrm{n}=3$. (B) Level of $\mathrm{p} 38$ and phosphorylated p38 (p-p38) protein in H9c2s treated with PBS, CpG-ODN, control CpG-ODN, SB203580, or CpG-ODN+SB203580 prior to I/R. ${ }^{*} \mathrm{P}<0.05$, ${ }^{* *} \mathrm{P}<0.01, \mathrm{n}=3$. I/R, ischemia/reperfusion; ODN, oligodeoxynucleotide; PBS, phosphate buffered saline. 


\section{Cellular Physiology Cell Physiol Biochem 2018;47:1389-1398

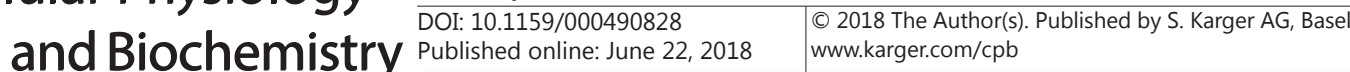

ODN contain unmethylated CpG motifs that activate TLR9 receptors. In the present study, we observed that administration of CpGODN increased the levels of phospho-p38 in the myocardium following myocardial I/R, indicating that CpG-ODN treatment activated the TLR9-p38 MAPK signaling pathway. Recently, two studies found that iCpG-ODN or TLR9 antagonist could reduce I/ $R$ damage in brain or liver $[16,17]$. Therefore, activating the TLR9 receptor is not beneficial.

TLR9's role in I/R is controversial. Previous studies have found that TLR9 activates the downstream PI3K pathway, and numerous studies have found that this is a protective pathway in cardiac muscle $[13,26$ 30]. However, TLR9 can also activate other signaling pathways, such as those found to have a role in myocardial injury.

To determine the mechanisms by which CpG-ODN treatment activated TLR9-p38 MAPK signaling, we examined whether there is an association between TLR9 and p38 following CpG-ODN administration. We performed in vitro experiments using $\mathrm{H} 9 \mathrm{c} 2$ cells and observed that CpG-ODN administration increased the levels of phospho-p38, while increased enzymatic activity of p38 was inhibited by SB203580. However, one limitation is that we did not further investigate p38 targets. Whether p38 phosphorylation was indeed reduced with SB203580 treatment or was unchanged is not clear. Despite these limitations, our data confirmed that CpG-ODNinduced cardiac injury is mediated through a TLR9-p38 MAPK-dependent mechanism.

In summary, administration of the TLR9 ligand CpG-ODN significantly increased I/Rinduced infarct size and reduced cardiac function following myocardial I/R.

\section{Acknowledgements}

The program is supported by grants from the National Natural Science Foundation of China (No. 81773963).

\section{Disclosure Statement}

The author declare that they have no conflict of interests.

\section{References}

1 Aderem A, Ulevitch RJ: Toll-like receptors in the induction of the innate immune response. Nature 2000;406:782-787.

-2 Johnson GL, Lapadat R: Mitogen-activated protein kinase pathways mediated by ERK, JNK, and p38 protein kinases. Science 2002;298:1911-1912.

-3 Latz E, Visintin A, Espevik T, Golenbock DT: Mechanisms of TLR9 activation. J Endotoxin Res 2004;10:406412. 


\section{Cellular Physiology Cell Physiol Biochem 2018;47:1389-1398 \begin{tabular}{l|l} 
and Biochemistry Published online: June 22, 2018 & $\begin{array}{l}\text { (c) } 2018 \text { The Author(s). Published by S. Karger AG, Basel } \\
\text { www.karger.com/cpb }\end{array}$
\end{tabular} \\ Xie et al.: Cpg-ODN Aggravates Myocardial Ischemia/Reperfusion Injury}

4 Kumagai Y, Takeuchi O, Akira S: TLR9 as a key receptor for the recognition of DNA. Adv Drug Deliver Rev 2008;60:795-804.

5 Krieg AM: Therapeutic potential of Toll-like receptor 9 activation. Nat Rev Drug Discov 2006;5:471-484.

6 Sester DP, Brion K, Trieu A, Goodridge HS, Roberts TL, Dunn J, Hume DA, Stacey KJ, Sweet MJ: CpG DNA activates survival in murine macrophages through TLR9 and the phosphatidylinositol 3-kinase-Akt pathway. J Immunol 2006;177:4473-4480.

7 Zhang Q, Raoof M, Chen Y, Sumi Y, Sursal T, Junger W, Brohi K, Itagaki K, Hauser CJ: Circulating mitochondrial DAMPs cause inflammatory responses to injury. Nature 2010;464:104-107.

-8 Chong AJ, Shimamoto A, Hampton CR, Takayama H, Spring DJ, Rothnie CL, Yada M, Pohlman TH, Verrier ED: Toll-like receptor 4 mediates ischemia/reperfusion injury of the heart. J Thorac Cardiovasc Surg 2004;128:170-179.

-9 Hua F, Ha T, Ma J, Li Y, Kelley J, Gao X, Browder IW, Kao RL, Williams DL, Li C: Protection against myocardial ischemia/reperfusion injury in TLR4-deficient mice is mediated through a phosphoinositide 3-kinasedependent mechanism. J Immunol 2007;178:7317-7324.

10 Li C, Ha T, Kelley J, Gao X, Qiu Y, Kao RL, Browder W, Williams DL: Modulating Toll-like receptor mediated signaling by (1-->3)-beta-D-glucan rapidly induces cardioprotection. Cardiovasc Res 2004;61:538-547.

11 Oyama J, Blais C, Jr., Liu X, Pu M, Kobzik L, Kelly RA, Bourcier T: Reduced myocardial ischemia-reperfusion injury in toll-like receptor 4-deficient mice. Circulation 2004;109:784-789.

12 Markowski P, Boehm O, Goelz L, Haesner AL, Ehrentraut H, Bauerfeld K, Tran N, Zacharowski K, Weisheit C, Langhoff P, Schwederski M, Hilbert T, Klaschik S, Hoeft A, Baumgarten G, Meyer R, Knuefermann P: Preconditioning with synthetic $\mathrm{CpG}$-oligonucleotides attenuates myocardial ischemia/reperfusion injury via IL-10 up-regulation. Basic Res Cardiol 2013;108:376.

13 Cao Z, Ren D, Ha T, Liu L, Wang X, Kalbfleisch J, Gao X, Kao R, Williams D, Li C: CpG-ODN, the TLR9 agonist, attenuates myocardial ischemia/reperfusion injury: involving activation of PI3K/Akt signaling. Biochim Biophys Acta 2013;1832:96-104.

$>14$ Zhou DC, Su YH, Jiang FQ, Xia JB, Wu HY, Chang ZS, Peng WT, Song GH, Park KS, Kim SK, Cai DQ, Zheng L, Qi XF: CpG oligodeoxynucleotide preconditioning improves cardiac function after myocardial infarction via modulation of energy metabolism and angiogenesis. J Cell Physiol 2018;233:4245-4257.

15 Kim SC, Wu S, Fang X, Neumann J, Eichhorn L, Schleifer G, Boehm O, Meyer R, Frede S, Hoeft A, Baumgarten G, Knuefermann P: Postconditioning with a CpG containing oligodeoxynucleotide ameliorates myocardial infarction in a murine closed-chest model. Life Sci 2014;119:1-8.

16 Zhou Y, Pan J, Peng Q Dong ZD, Deng LD, Wang Y: The TLR9 antagonist iCpG-ODN at different dosages inhibits cerebral ischemia/reperfusion injury in mice. CNS Neurol Disord-DR 2017;10.2174/18715273166 66170206150259

17 Shaker ME, Trawick BN, Mehal WZ: The novel TLR9 antagonist COV08-0064 protects from ischemia/ reperfusion injury in non-steatotic and steatotic mice livers. Biochem Pharmacol 2016;112:90-101.

18 Xie L, Liu S, Cheng J, Wang L, Liu J, Gong J: Exogenous administration of mitochondrial DNA promotes ischemia reperfusion injury via TLR9-p38 MAPK pathway. Regul Toxicol Pharm 2017;89:148-154.

19 Ha T, Hua F, Liu X, Ma J, McMullen JR, Shioi T, Izumo S, Kelley J, Gao X, Browder W, Williams DL, Kao RL, Li C: Lipopolysaccharide-induced myocardial protection against ischaemia/reperfusion injury is mediated through a PI3K/Akt-dependent mechanism. Cardiovasc Res 2008;78:546-553.

20 Sakata Y, Dong J-W, Vallejo JG, Huang C-H, Baker JS, Tracey KJ, Tacheuchi O, Akira S, Mann DL: Toll-like receptor 2 modulates left ventricular function following ischemia-reperfusion injury. Am J Physiol-Heart C 2007;292:H503-H509.

21 Hua F, Ha T, Ma J, Li Y, Kelley J, Gao X, Browder IW, Kao RL, Williams DL, Li C: Protection against myocardial ischemia/reperfusion injury in TLR4-deficient mice is mediated through a phosphoinositide 3-kinasedependent mechanism. J Immunol 2007;178:7317-7324.

22 Ha T, Hu Y, Liu L, Lu C, McMullen JR, Kelley J, Kao RL, Williams DL, Gao X, Li C: TLR2 ligands induce cardioprotection against ischaemia/reperfusion injury through a PI3K/Akt-dependent mechanism. Cardiovasc Res 2010;87:694-703.

23 Ha T, Lu C, Liu L, Hua F, Hu Y, Kelley J, Singh K, Kao RL, Kalbfleisch J, Williams DL, Gao X, Li C: TLR2 ligands attenuate cardiac dysfunction in polymicrobial sepsis via a phosphoinositide 3-kinase-dependent mechanism. Am J Physiol-Heart C 2010;298:H984-H991. 


\section{Cellular Physiology Cell Physiol Biochem 2018;47:1389-1398

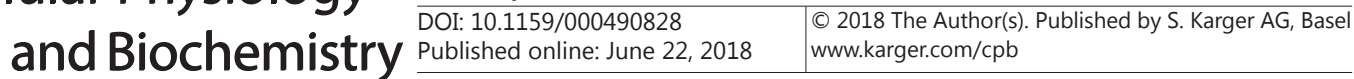 \\ Xie et al.: Cpg-ODN Aggravates Myocardial Ischemia/Reperfusion Injury}

24 Sun Z, Wang C, Shi C, Sun F, Xu X, Qian W, Nie S, Han X: Activated Wnt signaling induces myofibroblast differentiation of mesenchymal stem cells, contributing to pulmonary fibrosis. Int J Mol Med 2014;33:10971109.

25 Heusch G, Gersh BJ: The pathophysiology of acute myocardial infarction and strategies of protection beyond reperfusion: a continual challenge. Eur Heart J 2017;38:774-784.

26 Lu C, Ha T, Wang X, Liu L, Zhang X, Kimbrough EO, Sha Z, Guan M, Schweitzer J, Kalbfleisch J, Williams D, Li C: The TLR9 ligand, CpG-ODN, induces protection against cerebral ischemia/reperfusion injury via activation of PI3K/Akt signaling. J Am Heart Assoc 2014;3:e000629.

-27 Ha T, Hua F, Liu X, Ma J, McMullen JR, Shioi T, Izumo S, Kelley J, Gao X, Browder W, Williams DL, Kao RL, Li C: Lipopolysaccharide-induced myocardial protection against ischaemia/reperfusion injury is mediated through a PI3K/Akt-dependent mechanism. Cardiovasc Res 2008;78:546-553.

28 Zhu S, Xu T, Luo Y, Zhang Y, Xuan H, Ma Y, Pan D, Li D, Zhu H: Luteolin Enhances Sarcoplasmic Reticulum Ca2+-ATPase Activity through p38 MAPK Signaling thus Improving Rat Cardiac Function after Ischemia/ Reperfusion. Cell Physiol Biochem 2017;41:999-1010.

29 Al-Herz W, Babiker F: Acute Intravenous Infusion of Immunoglobulins Protects Against Myocardial Ischemia-Reperfusion Injury Through Inhibition of Caspase-3. Cell Physiol Biochem 2017;42:2295-2306.

30 Kang B, Li W, Xi W, Yi Y, Ciren Y, Shen H, Zhang Y, Jiang H, Xiao J, Wang Z: Hydrogen Sulfide Protects Cardiomyocytes against Apoptosis in Ischemia/Reperfusion through MiR-1-Regulated Histone Deacetylase 4 Pathway. Cell Physiol Biochem 2017;41:10-21. 